\title{
In vitro generation of platelets through direct conversion: first report in My Knowledge (iMK)
}

Cell Research (2013) 23:176-178. doi:10.1038/cr.2012.142; published online 9 October 2012

Blood transfusion medicine requires a constant supply of platelets, which is now totally donor dependent. Recent advances in the generation of platelets in vitro through megakaryocytes (MKs) may provide protocols not only via pluripotent stem cells, including induced pluripotent stem cells (iPSCs) and embryonic stem cells (ESCs), but also via induced MKs (iMKs). For the first time, mouse and human fibroblasts are successfully transdifferentiated into iMKs by the introduction of three factors, p45NF-E2, Maf G, and Maf K.

Platelets are essential for hemostasis, and adhesive functions of platelets play key roles in stopping bleeding [1]. Patients with thrombocytopenia receive platelet transfusions, which are supplied by donors. A donor-dependent supply of platelets is clearly unstable; furthermore, platelets are known to have a short shelf life because they cannot be frozen and should be used within 3-4 days after collection. In order to reduce the total amount of platelets needed for transfusion into patients, a new strategy has recently been proposed; it is a "therapeutic strategy", in which patients receive a platelet transfusion when bleeding occurs, compared with the conventional "prophylactic strategy", in which patients receive a platelet transfusion when platelet counts are below the threshold [2]. This study clearly reflects the seriousness of an insufficient and unstable supply of platelets, despite a long-term pursuit of a donor-independent system.

Recently, Matsubara and colleagues
[3] have demonstrated that mouse and human fibroblasts can be directly converted into megakaryocytes (MKs), from which platelets are released. The authors identified three factors as MKinducing factors, i.e., p45NF-E2, Maf G, and Maf K (Figure 1). How did they find these factors? In their previous studies, they obtained abundant MKs and platelets from human subcutaneous adipose tissues [4], which were cultured in megakaryocyte lineage induction (MKLI) medium. MKLI medium, which was also used in the present research [3], contained thrombopoietin (TPO). Furthermore, they examined the ability of mouse fibroblast 3T3 cells and 3T3-L1 cells (preadipocyte cell line) to differentiate into the MK lineage [5]. They clearly showed in vitro differentiation potential of 3T3-L1 cells, but not of $3 T 3$ cells, into the MK lineage. In the present research [3], to screen for MK-inducing factors, gene expressions were compared between 3T3-L1 and $3 \mathrm{~T} 3$ cells. The candidate genes were p45NF-E2 and CEBP- $\alpha$, and the latter is a determinant adipocyte marker. They also selected Maf G and Maf $\mathrm{K}$, which are known binding partners of p45NF-E2 [6]. Among the several combinations tested, transfection of p45NF-E2/Maf G/Maf K into mouse and human fibroblasts led to the highest frequency of cells that were positive for CD41, a marker for MKs and platelets. When adult human dermal fibroblasts (HDFs) were examined, more than $90 \%$ of the MK-sized cells expressed CD41, and were thus designated induced MKs (iMKs) (Figure 1). It took only 2 weeks to obtain iMK cells (from which platelets are released), which is more rapid than other methods using induced pluripotent stem cells (iPSCs) or embryonic stem cells (ESCs). However, iMK cells in this system yield fewer platelets (510 platelets per iMK cell) than MKs do in vivo, where 2000 platelets can be generated per MK.

On the other hand, iPSC- or ESCmediated generation of platelets in humans has been demonstrated by Eto and colleagues $[7,8]$. They revealed that a transient activation of c-Myc during megakaryopoiesis is critical for efficient platelet production from human iPSCs [8] (Figure 1); that is, reactivation of c-Myc after reprogramming should be followed by reduction of $\mathrm{c}-\mathrm{Myc}$ expression for further maturation. In contrast, sustained and excessive c-Myc expression in MKs was accompanied by increased p14 (ARF) and p16 (INK4A) expression, and decreased GATA1 and NF-E2 expression, eventually leading to MK senescence and apoptosis, as well as impaired production of functional platelets [8]. It took approximately 2 months to obtain MKs by reprogramming HDFs into iPSCs and then by differentiating iPSCs into MKs. More recently, in order to obtain large numbers of platelets rapidly, Eto and colleagues have established an immortalized MK cell line (MKCL) derived from human iPSCs [9] (Figure 1). The immortalized MKCL could potentially provide a stable supply of high quality platelets for transfusion medicine.

Now, we can compare iMKs with iPSC-mediated MKs or with MKCLs on 
several points. i) Regarding cell numbers, Matsubara and colleagues obtained $8-10 \times 10^{5}$ iMKs from $20 \times 10^{6} \mathrm{HDFs}$ [3]. Although HDFs are easy to expand once direct conversion is initiated, the number of transfected cells is limited. Eto and colleagues generated 200-300 iPS clones from $10^{5} \mathrm{HDFs}$, and $1 \times 10^{5}$ cultured iPSCs produced approximately $17 \times 10^{5} \mathrm{MKs}$ [8]. Although it is difficult to precisely compare reprogramming efficiency between the iMK study and the iPSC study, the number of platelets per MK was similarly low in both cases, which may reflect the inefficiencies of producing human platelets via in vitro protocols. ii) With regard to the time required for the production of MKs and platelets, iMKs and iPSC-mediated MKs are induced in approximately 2 weeks and 2 months, respectively. MKCLs may represent a readily accessible source for platelet production. iii) In terms of risk of contamination of undifferentiated cells, although iPSC-mediated MKs may contain some residual undifferentiated cells, it will be safe to transfuse platelets from these MKs, because platelets (anucleated cells) may be irradiated before transfusion. iv) Regarding cryopreservation, iPSCs and MKCLs are immortal cell lines and easy to freeze down. If HDFs are used as starting materials for iMK induction, HDFs are able to be expanded to some extent and are suitable for cryopreservation. Platelets are known to be unsuitable for cryopreservation. v) With regard to functionality, Matsubara and colleagues [3] infused iMKs into irradiated NOG mice to examine whether iMKs can release platelets in vivo. Tail vein blood samples were obtained around $3 \mathrm{~h}$ after iMK infusion, and it was demonstrated that iMKs released human platelets in vivo with an estimated 5-10 platelets per infused iMK cell. To examine whether iMKderived platelets are functional, ex vivo thrombus formation under flow condition was also tested, and functionality was confirmed [3]. As demonstrated

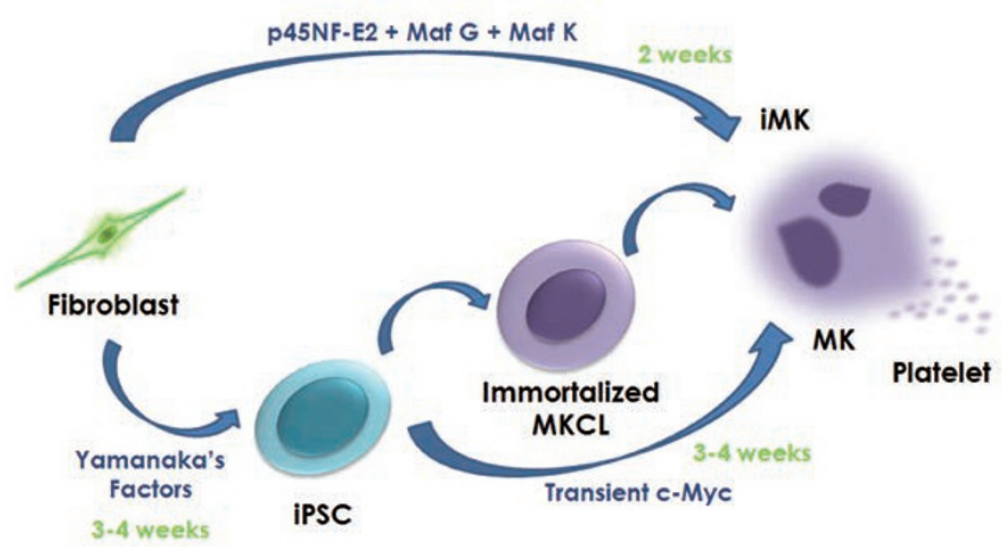

Figure 1 Schematic diagrams of MK induction from human dermal fibroblasts. i) Direct conversion (iMK) method: three factors (p45MF-E2, Maf G, and Maf K) are required for direct conversion into iMK, which takes only 2 weeks [3]. ii) iPSCmediated method: transient activation of c-Myc in iPSCs is required for efficient MK induction [8]. If megakaryocyte progenitor cells are immortalized (designated as MKCLs), MKCLs are valuable sources for MK production [9]. MK, megakaryocyte; iMK, induced megakaryocyte; iPSC, induced pluripotent stem cell; MKCL, megakaryocyte cell line.

in previous papers $[8,10]$, it should be proved in the future that iMK-derived platelets circulate in NOG mice and are able to form thrombus in vivo.

iMKs have been reported for the first time, and may provide us valuable options for transfusion medicine. However, there remain some concerns to be addressed.

First, the molecular mechanism by which iMKs are induced through the p45NF-E2 transcriptional complex has yet to be elucidated. Moreover, whether p45NF-E2, Maf G, and Maf K represent the optimal factor combination should be investigated. Key regulators of MK maturation, such as GATA1 and $\beta 1$ tubulin, were checked, but were not actually transduced into HDFs [3]. Based on the findings by Eto and colleagues, it would be interesting to examine the role of transient expression of c-Myc and/or BMI1 (negative regulator for both $I N K 4 A$ and $A R F$ genes) [9] in iMK induction.

Second, what kinds of cells are suitable for direct conversion into iMKs (or for establishment of MKCLs via iPSCs)? For example, the variability in hepatic differentiation of different
iPSC lines depends on the genetic background of the donor rather than the starting cell type [11]. On the contrary, other studies suggested the effect of epigenetic memory on differentiation potential [12]. If epigenetic memory is also important for iMK induction, peripheral blood cells would be useful, and they have the added advantage of being easily accessible. However, they are more difficult to expand than HDFs. Thus, further improvements are needed for direct conversion of white blood cells into platelets. Another candidate for starting samples would be adipose tissues; as mentioned above, human subcutaneous adipose tissues are the original source for MK induction [4], and $\mathrm{MK}$ induction might be enhanced by the addition of the three factors. If cells responsible for $\mathrm{MK}$ induction in the conventional context [4] could be isolated and identified among human adipose tissues, and if immortalized cell lines could be established from those cells, it may be possible to induce MKs more efficiently with or without three factors from human adipose-derived immortalized cell lines.

Finally, is it necessary to generate 
autologous iMKs through direct conversion? In general, platelet transfusion does not require matching of human leukocyte antigen (HLA) between the donor and recipient; ABO-identical (non-HLA-typed) platelets are enough for transfusions. Although it is relatively rare, repeated platelet transfusions would result in undesired production of antibodies against HLA or human platelet antigen (HPA) on transfused platelets, which could lead to refractoriness to platelet transfusion unless HLA-matched platelets are transfused [1]. In such cases, it takes much effort and time to prepare HLA-matched platelets from donors to avoid immune rejections mediated by antibodies against HLA or HPA. Autologous iMKs through direct conversion could resolve this problem. Another way to resolve this issue is to utilize iPSC banks, in which homozygous HLA-typed iPSCs would be deposited for HLA-matched tissue transplantation [13]. It was shown that a tissue bank from 150 selected homozygous HLA-typed volunteers could match $93 \%$ of the UK population [13]. Similarly, Yamanaka and colleagues calculated that 140 unique HLA homozygous donors could provide a match for $90 \%$ of the Japanese population [14]. Additionally, it might be a practical approach to use existing HLA-typed samples, such as those in a bone-marrow-donor bank or in a cord- blood bank. More recently, Yamanaka and his team [15] revealed their new plan to generate 75 iPSC lines from HLA homozygous cord-blood cells, which will provide a match for $80 \%$ of the Japanese population. Moreover, if MKCLs are established from each homozygous HLA-typed iPSC, this could contribute to the resolution of unresponsiveness to platelet transfusion.

In summary, to our knowledge, the study by Matsubara and colleagues [3] is the first report of iMKs, which are directly converted from mouse and human fibroblasts. Although the precise mechanism of iMK induction is poorly understood, and efficient platelet release remains to be achieved, recent progress opens up a new era in platelet production in vitro. Since hematopoietic stem cells are difficult to maintain or expand in vitro, either direct conversion into MKs or directed differentiation to MKs from iPSCs/ESCs (or MKCLs) can be potential options for cultured platelets (Figure 1). Platelets contain no nucleus (anucleate) and are not affected by genetic manipulations, which would suggest no risk of transformation and would be an advantage for future clinical trials, while platelet production from MKCLs might also be relatively close to clinical trials.

Shigeo Masuda ${ }^{1}, \mathrm{Mo} \mathrm{Li}^{1}$, Juan Carlos Izpisua Belmonte ${ }^{1,2}$
${ }^{I}$ Gene Expression Laboratory, Salk Institute for Biological Studies, 10010 North Torrey Pines Road, La Jolla, CA 92037, USA; ${ }^{2}$ Center for Regenerative Medicine in Barcelona, Dr. Aiguader 88, 08003 Barcelona, Spain

Correspondence: Juan Carlos Izpisua Belmonte Tel: +1-858-453-4100; Fax: +1-858-453-2573

E-mail: belmonte@salk.edu, izupisua@cmrb.eu

\section{References}

1 Stroncek DF, Rebulla P. Lancet 2007; 370:427-438.

2 Wandt H, Schaefer-Eckart K, Wendelin K, et al. Lancet 2012; 380:1309-1316.

3 Ono Y, Wang Y, Suzuki H, et al. Blood 2012; 120:3812-3821.

4 Matsubara Y, Saito E, Suzuki H, et al. Biochem Biophys Res Commun 2009; 378:716720 .

5 Matsubara Y, Suzuki H, Ikeda Y, et al. Biochem Biophys Res Commun 2010; 402:796800.

6 Igarashi K, Kataoka K, Itoh K, et al. Nature 1994; 367:568-572.

7 Takayama N, Nishikii H, Usui J, et al. Blood 2008; 111:5298-5306.

8 Takayama N, Nishimura S, Nakamura S, et al. J Exp Med 2010; 207:2817-2830.

9 Nakamura S, Takayama N, Nakauchi H, et al. Blood 2011; 118:2.

10 Lu SJ, Li F, Yin H, et al. Cell Res 2011; 21:530-545.

11 Kajiwara M, Aoi T, Okita K, et al. Proc Natl Acad Sci USA 2012; 109:12538-12543.

12 Kim K, Doi A, Wen B, et al. Nature 2010; 467:285-290.

13 Taylor CJ, Peacock S, Chaudhry AN, et al. Cell Stem Cell 2012; 11:147-152.

14 Okita K, Matsumura Y, Sato Y, et al. Nat Methods 2011; 8:409-412.

15 Cyranoski D. Nature 2012; 488:139. 